\title{
Despersonalización de los medios, poder y guerras de audiencia en el retrato del periodismo en el cine de los años 90
}

\author{
Olga OSORIO IGLESIAS \\ Universidad de A Coruña \\ olga.osorio@udc.es
}

Recibido: 22 de julio de 2013

Aceptado: 21 de enero de 2014

\begin{abstract}
Resumen
La lectura global del retrato que el cine de los 90 ofrece de los medios de comunicación a partir de un repaso de los elementos más repetidos en su representación cinematográfica permite concluir que los elementos más destacados por el cine de la década son el poder y la presencia de los medios de comunicación en todos los ámbitos de la vida cotidiana, así como su despersonalización, reflejada en la nube de periodistas y su acoso a los protagonistas de la información. El estudio se basa en el análisis de 112 películas estrenadas entre 1990 y 1999 y forma parte de un proyecto más amplio del que surge la tesis doctoral "La imagen de la periodista profesional en el cine de ficción de 1990 a 1999".

Palabras clave: periodismo, cine, imagen de los periodistas en la sociedad, años 90 , medios de comunicación

\section{Depersonalization of media, power and the battle for audiences in the portrait of journalism in the movies of the $90 \mathrm{~s}$}

\begin{abstract}
An overall reading of the film portrait of the media in the 90s, from a review of the most repeated elements in the representation, leads to conclude that the cinema of the decade highlights the power and presence of the media in all areas of daily life and its depersonalization, reflected in the swarm of reporters and the way they harass the main protagonists of the information. The corpus of the analysis is composed by 112 films released between 1990 and 1999 and is part of a larger project in which was born the $\mathrm{PhD}$ dissertation "The image of the professional journalist fiction films from 1990 to 1999."
\end{abstract}

Keywords: journalism, cinema, journalist image in society, 90s, media

\section{Referencia normalizada}

OSORIO IGLESIAS, Olga (2014): "Despersonalización de los medios, poder y guerras de audiencia en el retrato del periodismo en el cine de los años 90". Estudios sobre el Mensaje Periodístico. Vol. 20, Núm. 2 (julio-diciembre), págs.: 789-804. Madrid, Servicio de Publicaciones de la Universidad Complutense.

Sumario: 1. Introducción. 2. Metodología y fuentes. 3. El periodismo en el cine de los años 90: datos cuantitativos. 4. Análisis de la representación. 5. Conclusiones. 6. Referencias bibliográficas. 7. Anexo: Películas de los años 90 en las que aparecen periodistas.

\section{Introducción}

El tratamiento de los periodistas en el cine del siglo XX es la historia de la fluctuación entre su consideración héroes o como villanos (Ehrlich, 2004) (Ghiglione \& Saltzman, 2002) (McNair, 2010). Los años 90 ofrecen una imagen del periodista especialmente beligerante, que ha sido abordada ya con mayor o menor profundidad en algunos trabajos específicos (Steinle, 2000) (Ehlers, 2006) (Rosa, 2006) (Osorio, 2009). Sin embargo, es mucho menos habitual el estudio global de la representación cinematográfica de la relación entre el periodismo y la sociedad, más allá del análisis 
del estereotipo del periodista cinematográfico o del quehacer profesional de los periodistas. Lo que se propone en este artículo es, en suma, una revisión global del modo en que el cine retrata el comportamiento de los periodistas como institución y su relación con la sociedad, partiendo de la base de que las creencias de los ciudadanos sobre la realidad que los rodea está bastante condicionada por el retrato que los medios de comunicación hacen de ella. De acuerdo con la teoría del cultivo acuñada por George Gebner, los medios ofrecen una imagen de la sociedad bastante coherente y repetitiva que no responde a la realidad pero que condiciona en gran medida las creencias que sobre ella posee el público, en mayor medida cuanto más expuesto está a dichos mensajes (Morgan, 2008). La teoría sobre los usos y gratificaciones (Sherry \& Boyan, 2008) lleva a suponer -a partir del influyente estudio de 1974 de Katz, Blumler y Gurevitch- que la propuesta mediática ha de guardar bastante afinidad con las ideas preconcebidas de los espectadores.

\section{Metodología y fuentes}

El análisis propuesto se basa en el visionado de 112 películas estrenadas en los años 90 en cine en España en las que aparecen periodistas en roles significativos (Osorio, 2009, pp. 473-481). Para elaborar dicho listado se llevó a cabo una consulta exhaustiva de la bibliografía específica sobre cine y periodismo, en especial, aunque no exclusivamente, el exhaustivo trabajo de Richard Ness, que cubre desde los orígenes del cine hasta 1996 (Ness, 1997). Otros autores consultados fueron: (Mínguez Santos, 2012) (Laviana, 1996) (García de Lucas, et al., 2006) (Bezunartea, et al., 2007) (Bezunartea, et al., 2007) (Bezunartea, et al., 2008) (Bezunartea, et al., 2008) (Bezunartea Valencia, et al., 2010) (Ghiglione, 1991) (Bezunartea Valencia, et al., 2010) (Good, 1998) (Herman, 2004-2005) (Robards, 1990) (Saltzman, 2003). Adicionalmente se llevó a cabo un vaciado de otros volúmenes y artículos sobre cine como Guía del cine español (Aguilar, 2007), 100 películas de cine para trabajar la televisión en el aula (PardoAlarcón Castellón, 2000) y la guía publicada en 2009 por Leonard Maltin (2008).

La consulta a cuatro bases de datos (Image of Journalist in Popular Culture, 2013) (Anon., 2013) (Anon., 2013) (Ministerio de Educación, Cultura y Deportes, 2013), unida a la bibliografía específica mencionada, constituyó el principal recurso. En el caso de la base de datos del grupo de investigación Image of Journalist in Popular Culture, su estructura, sin apenas división en campos ni utilización de palabras clave, dificultó la selección entre los más de quince mil títulos fílmicos que incluye, muchos de ellos obtenidos a su vez a través de un vaciado de IMDB, lo que hace que en muchos filmes aparezcan paradójicamente mencionados los periodistas que ocupan papeles secundarios pero no los protagonistas ${ }^{1}$. Pese a la dificultad de su manejo y a las

1 Un solo ejemplo de entre los muchos que se podrían poner: IJPC indexa Sostiene Pereira (Roberto Faenza, 1996) incluyendo sólo en el campo de "comentarios" (un campo genérico en el que se incluye cualquier tipo de información sobre el film) al director del periódico, un personaje muy secundario, gracias a que el mismo está identificado como tal en IMDB, sin mencionar, por tanto, que el protagonista es un periodista y que la película aborda cuestiones cruciales de la profesión. 
carencias comentadas, IJPC database constituye una fuente imprescindible para cualquier trabajo sobre la imagen de los periodistas en la cultura popular, así como un inestimable punto de partida.

La base de datos del Ministerio de Educación, Cultura y Deportes sirvió a su vez como filtro final para confirmar si el film había sido estrenado o no en España y para incorporar alguna información que se consideró relevante, como el número de espectadores obtenidos por cada uno de los filmes, aparte de cómo fuente principal para muchas películas de producción española.

Una última fuente para la elaboración de este listado fueron páginas web que ofrecen recopilaciones semejantes, elaboradas, en su caso, con una finalidad divulgativa. Entre estos sitios web figura la sección dedicada al "Periodismo y los Medios de Comunicación en el Cine" (Martínez-Salanova Sánchez, 2011) de un sitio más amplio que el autor ha creado como soporte de material didáctico diverso. Asimismo, se ha tomado también como referencia, sobre todo a la hora de considerar si una cinta pertenecía o no al subgénero "periodismo", la selección elaborada por Paul E. Schindler Jr. (1996). Otros sitios web revisados son About.com (Deahl, s.f.) y, sobre todo, el interesantísimo repertorio bibliográfico y filmográfico publicado en la red por la biblioteca de la Universidad de Berkeley (University of California, Berkeley, 1996-2009).

\section{El periodismo en el cine de los años 90: datos cuantitativos}

El vaciado de las fuentes anteriormente citadas permitió, como ya se apuntó, generar una filmografía integrada por 112 películas en las que aparece algún periodista en papeles de cierta relevancia. Se excluyeron los títulos en los que los periodistas localizados lo hacían en calidad de extra sin frase, así como los de muy difícil acceso en la actualidad $^{2}$ y que tuvieron además escasa distribución también en el momento de su estreno $^{3}$. Se excluyeron también las películas en las que los periodistas no ejercían como tales de forma profesional y remunerada, de modo que atendiendo a esta premisa quedaron al margen de la investigación los personajes que pertenecen a colectivos como estudiantes o profesores los aspirantes a profesionales, aunque ya hayan acabado sus estudios y los que ejercen actividades periodísticas de forma no remunerada como, por ejemplo, en publicaciones estudiantiles o a través de Internet, así como aquellos

2 The Crush (Veneno en la piel), (Alan Saphiro, 1993); Vivir así (Luis Martínez Rodríguez, 1999); Adão e Eva (Adán y Eva), (Joaquim Leitão, 1995); Sur La terre Comme Au Ciel (Entre el cielo y la tierra), (Marion Hänsel, 1992), Turtle Beach (Stephen Wallace, 1992); Livin'Large! (Reportero por los pelos), (Michael Schultz, 1991); Cobb (Ty Cobb), (Ron Shelton, 1994); La hermana (Juan José Porto, 1995); Luz negra (Xavier Bermúdez, 1995); Entre todas las mujeres (Juan Ortuoste, 1997); A solas contigo (Eduardo Campoy, 1990); Cover Up (Rescate) (1990); The Innocent Sleep (El sueño inocente) (Scott Michell, 1995), V.I. Warshawski (Detective con medias de seda), (Jeff Kanew, 1991); Atilano, presidente (La Cuadrilla, 1990).

3 De acuerdo con los datos obtenidos de la base de datos del Ministerio de Educación, Cultura y Deportes. 
que de forma esporádica y puntual ejercen como periodistas, sin que ésta sea su actividad principal.

Hay también que apuntar que el objetivo de este estudio es clarificar cuál es la imagen que se ofrece del periodismo y no en general de los medios de comunicación, debido a lo cual no se consideran tampoco algunos títulos muy conocidos como $R e$ ality Bites (Bocados de realidad), (Stiller, 1994), en la que el personaje protagonista, interpretado por Winona Ryder, trabaja como asistente de producción en un talk-show, lo que hace que este film retrate el mundo de los medios de comunicación (fundamentalmente la televisión) pero no desde la perspectiva de la información sino desde el entretenimiento. También fueron excluidas The Truman's show (El show de Truman), (Peter Weir, 1998) y EdTV (Howard, 1999) cuyo tema es, en ambos casos, la relación entre la realidad y los medios de comunicación a través de una ficción sobre la “telerrealidad", Otra película descartada fue Quiz show (El dilema), (Robert Redford, 1994), que trata sobre el fraude en un concurso televisivo de temas culturales, en la que no aparecen periodistas.

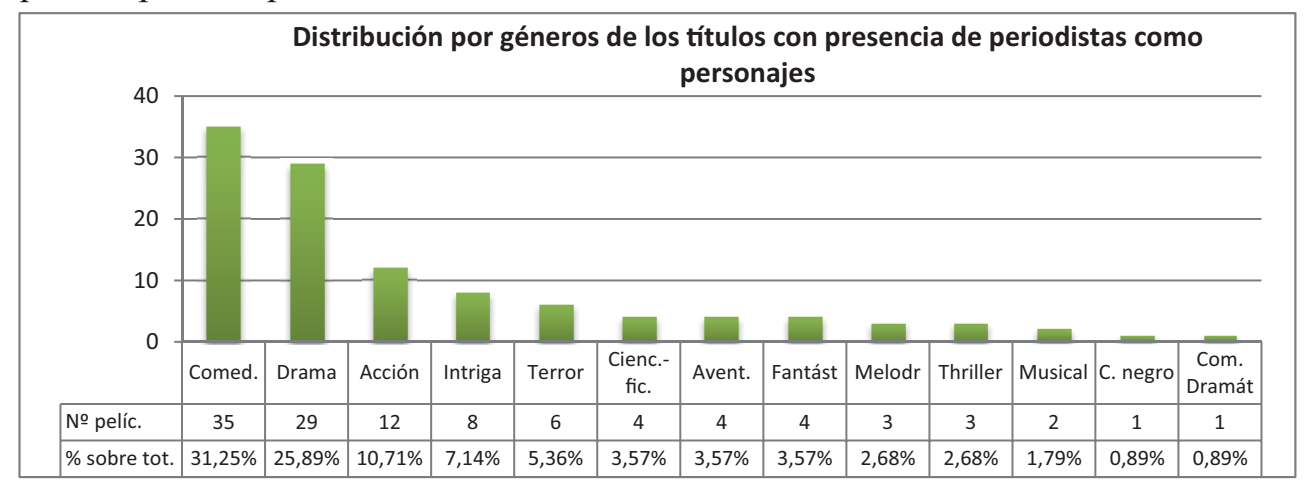

Gráfico 1. Distribución por géneros de las 112 películas con periodistas en roles significativos estrenadas en cine en España en los años 90. Fuente: elaboración propia

Una primera revisión de los 112 títulos permite detectar que las películas de la década en las que más predomina la presencia de periodistas son las comedias con un total de 35 títulos adscritos al mismo (31,25\%), a los que habría que sumar uno más encuadrado dentro de las "comedias dramáticas". Le sigue el drama, con $29(25,89 \%)$ $\mathrm{y}$, a continuación, las cintas de acción, con doce (10,71\%). Un género tan vinculado en sus orígenes con el periodismo como fue el cine negro muestra una presencia apenas testimonial en los años 90 con tan sólo una representación $(0,89 \%)$ y también decae en esta época la presencia de periodistas en títulos de intriga, a los que estuvieron muy asociados en los años dorados de la representación cinematográfica de los periodistas como fueron los años 30 y los 70 .

Por otra parte, entre 1990 y 1999 se estrenan trece películas en las que el periodismo y los periodistas constituyen el elemento fundamental, cuyo estudio exhaus-

4 Atendiendo a estos criterios se excluyen los siguientes títulos: Urban Legend (Leyenda urbana), (Jamie Blanks, 1998) y Tesis (Amenábar, 1996) 
tivo puede resultar de interés con objeto de determinar cuáles son las cuestiones que la industria cinematográfica considera cruciales en la década cuando plantea de manera expresa retratar el periodismo. Sin embargo, en este artículo la mirada pretende ser más amplia y se toman en consideración no sólo estas trece películas en particular sino, como ya se apuntó, todos los títulos de la década en los que aparece algún periodista en un rol dramático destacado. El objetivo es detectar si existe una representación más o menos reiterada del papel de los medios de comunicación en general y no de un determinado periodista en particular, ya sea a través de la puesta en escena, ya de los comentarios realizados por algún personaje integrado en la trama.

\section{Análisis de la representación}

Uno de los primeros aspectos detectados al analizar cuál es la imagen que ofrece el cine de los años 90 sobre el periodismo y los medios de comunicación es la clara consciencia de su importancia decisiva como canalizadores de la casi omnipotente "opinión pública". Lo que los medios difunden y sostienen es lo que los ciudadanos opinan, aunque en muchos casos se aleje bastante de la verdad. Ello hace que muchas películas muestren cómo desde la economía, la política o las propias empresas informativas hay una clara conciencia de la enorme influencia de los medios en el público, que no en no pocas ocasiones intentan manejar en provecho propio. Por otra parte, a finales de siglo los medios de masas, sobre todo la televisión, se han consolidado como parte indisociable de la vida de las personas, de ahí que en las películas de la última década del siglo XX los periodistas aparezcan en todas partes, no ya en la forma del periodista duro, solitario y comprometido con la verdad, sino encarnados en una nube anónima, agresiva y deshumanizada.

Tres películas producidas en dicha década sirven para ilustrar, de modo muy resumido y esquemático, la evolución de la interacción de los medios con sus fuentes en distintas épocas. En primer lugar, un par de secuencias de La ley de la frontera (Adolfo Aristarain, 1995) escenifican lo singular que resultaba la presencia de un periodista (una fotógrafa del New York Times, en este caso) en la Galicia de los años 20. Bárbara Miller Molina (Aitana Sánchez-Gijón) viaja en solitario por la frontera galaico-portuguesa en busca del "Argentino", un mítico bandolero al que quiere entrevistar en exclusiva. Ajena a las presiones del tiempo o de la febril competencia que caracterizará el trabajo de los periodistas en épocas más recientes, Bárbara aprovecha para hacer retratos costumbristas de las personas y situaciones que va encontrando a su paso, para lo que cuenta no sólo con la plena colaboración de los interesados sino también a menudo con una notable expectación.

Ambientada bastantes años más tarde, en 1978, Year of the gun (El año de las armas), (John Frankenheimer, 1991), pone en escena una relación de los medios con sus fuentes bastante distinta a la que predominará en el retrato de los 90. Así, el filme escenifica cómo los medios de comunicación abordan al primer ministro Aldo Moro a su llegada al aeropuerto de Roma. Aunque hay bastantes medios de comunicación presentes, todos mantienen una respetuosa y cómoda distancia con el entrevistado (sólo en un momento se ve, al fondo del plano, cómo un cámara intenta acercarse demasiado al primer ministro desde atrás, un gesto impedido por un miembro del equipo 
de seguridad). Sobre todo resulta significativa la calma con la que los periodistas abordan su trabajo, que incluso permite a la periodista de la BBC, interpretada por Cecilia Todeschini, realizar una pequeña entrevista con coherencia y sin ser constantemente interrumpida por sus compañeros, algo impensable en las escenificaciones de la "nube de periodistas" características de las películas ambientadas en una época contemporánea a la de su producción.

En efecto, los periodistas retratados en general por el cine de los años 90 se presentan como un grupo desorganizado, compacto y ruidoso que corre enloquecido en pos de su fuente de información. Su elevado número los deshumaniza, dificulta su trabajo e intimida a sus entrevistados, cuya relación con los medios, al menos cuando se presentan como grupo, cambia de la colaboración que se reflejaba en las secuencias anteriores al rechazo o incluso a los intentos de huida. No es raro que este tipo de escenas terminen con el personaje acorralado contra una puerta, pared o similar con los periodistas disparándole preguntas y flashazos.

Son muchas las películas en las que pueden verse escenas similares, de modo que me detendré sólo en las más llamativas. Una de ellas es el momento en que Sam Baily (John Travolta) libera en Mad City (Constantin Costa-Gavras, 1997) a dos de las escolares del grupo al que retiene como rehenes en un museo en protesta por su despido. La salida de las niñas, en el minuto 33 de la película, es espectacular. En plano las muestra descendiendo, temerosas, por una escalera, mirando aterrorizadas hacia el frente. Lo que les causa pavor está en el fuera de campo, pero no tarda en entrar en plano: son los periodistas, que se acercan como una horda implacable ante la que las niñas retroceden asustadas, hasta que la policía interviene, cogiéndolas en brazos (hacer retroceder a los medios está claro que no es una opción).

No es la única escena de este tipo en la película, en la que se recogen varios momentos similares hasta llegar al estremecedor plano final, el congelado de un plano cenital al que se llega mediante un travelling de alejamiento que permite contemplar al periodista Max Brackett (Dustin Hoffman) rodeado por sus compañeros, que inundan todo el espacio visible.

En The Bonfire of Vanities (La hoguera de las vanidades), (Brian de Palma, 1990) también tiene un lugar destacado el grupo deshumanizado que su colega Peter Fallow (Bruces Willis) bautiza como "chacales". Aparecen en varias ocasiones en la película, la primera en el momento de máxima gloria personal y profesional Fallow con que se abre y cierra el filme. Aun cuando en este caso su presencia es "amistosa" (Fallow acude ante los medios para presentar su exitosa novela, ganadora de muchos premios), su presencia resulta intimidante incluso para uno de los suyos -al verlos correr hacia el personaje uno teme, por momentos, que no se detengan y lo arrollen, una impresión similar a la recibida en Mad City-. Con este punto de partida es fácil imaginar cuán agresivos resultan para Sherman McCoy (Tom Hanks), un yuppie de Wall Street acusado de atropellar a un joven negro del Bronx y salir huyendo y ya juzgado en los medios de comunicación como culpable. McCoy observa aterrorizado, desde el interior del coche, la nube de periodistas que lo asalta a su llegada al juzgado. En los planos tomados desde el exterior del vehículo, los representantes de los medios, que se resguardan de la lluvia bajo paraguas negros, se asemejan a una bandada de cuervos agresivos. 
Esta manera de abordar las fuentes noticiosas difícilmente puede facilitar el trabajo de los informadores. De hecho, estas secuencias nos muestran a los protagonistas de la información huyendo de los medios en vez de dialogando con ellos, de tal modo que tener la imagen del personaje, aunque sea escapando, se convierte en más importante que el propio contenido de la noticia. Al mismo tiempo, esta manera de encarar el trabajo informativo genera también una uniformización de la información (o de la falta de ella) suministrada al público. El mayor número de medios de comunicación no se traduce así en una mayor pluralidad informativa o en una disponibilidad de nuevos y variados puntos de vista sino que multiplica hasta la exageración un punto de vista único y con frecuencia poco cercano a la realidad sobre la que se pretende informar. Tal parece ser la reflexión que se impone tras el visionado en Deep Impact (Mimi Lerder, 1998) de un plano muy significativo ante la Casa Blanca, en el que un montón de los reporteros de televisión de medios diferentes hacen una entradilla al mismo tiempo, en el mismo lugar y es de suponer que con contenidos casi idénticos. Ante semejante uniformidad el espectador puede preguntarse cuál es el sentido de que haya tantos y tan variados medios presentes.

La nube de periodistas anónimos, aunque menos amenazadora en este caso, también aparece en The Pelican Brief, en la que los medios hacen guardia bajo la ventana del hospital en el que se hace su revisión médica anual el presidente de los Estados Unidos, interpretado por Robert Culp. En esta película, cuya visión del cuarto poder es mucho más positiva que en las repasadas hasta aquí, se refleja su papel como vigilantes de los políticos y de los poderes económicos. De hecho, el propio presidente en varias ocasiones alude a lo que ocurriría si el asunto destapado por el "informe pelícano" llegara a conocimiento de los medios, lo que en principio parece preocuparle más que la eventualidad de que llegue a manos de la Justicia, mientras que en el minuto 85.49 , se hace una breve referencia a los intentos por parte de los poderes públicos de utilizar la prensa a su favor, ya que se ve cómo los asesores del presidente planean filtrar un informe a los medios. Tampoco éstos están libres de críticas en este filme, ya que, por ejemplo, en el minuto 101.41 el padre de una de las víctimas de la trama conspiratoria que investigan le pregunta al protagonista, con acritud, desde cuándo la prensa respeta la intimidad de las personas.

El poder de los medios y su enorme influencia en la sociedad contemporánea aparece en numerosas películas en la década. En In\&Out (Frank Oz, 1997) la apacible vida de un profesor de Instituto se verá alterada por la repercusión casi global que obtiene la afirmación de que es gay proferida por uno de sus antiguos alumnos, ahora famoso actor de Hollywood, durante la gala de entrega de los Oscar. El comentario provoca varios efectos inmediatos, entre los cuales figura el aterrizaje en el hasta entonces apacible pueblo, de un nutrido grupo de periodistas (otra vez la nube deshumanizada) que acorrala a Howard Brackett (Kevin Kline) con su agresividad característica (minuto 00:18:00 del filme). Entre ellos, aunque distanciado del resto del grupo, se encuentra Peter Malloy (Tom Selleck), quien resume así la situación para las cámaras: "A teacher in trouble. A town under siege. A journey to the heartland. Stay tuned"5 (In\&out, 00:18:39 a 00:18:44).

5 "Un profesor en apuros, una ciudad sitiada, un viaje al corazón del país: permanezca en sintonía" (In\&out, 00:18:39 a 00:18:44). 
Una situación similar -el pequeño pueblo cuya tranquilidad se ve alterada sin remedio por una información de carácter personal e individual publicada en un medio de comunicación- también se retrata en Runaway Bride (Novia a la fuga), (Garry Marshall, 1999) o, aunque en este caso no es un pueblo sino un estadio de fútbol, en Never Been Kissed (Nunca me han besado), (Raja Gosnell, 1999).

Mucho más positiva es la influencia de los medios en la vida del joven Will Stoneman (MacKenzie Astin), al que la prensa bautiza como "Iron Will" (Voluntad de hierro) durante su participación en una durísima carrera de trineos convirtiéndolo así en un auténtico héroe y en un modelo para los jóvenes norteamericanos que acuden a prestarle su apoyo tras haber leído su historia en los diarios. Claro que los hechos que relata Iron Will (Voluntad de hierro), (Charles Haid,1994) tienen lugar a principios del siglo XX y que, además, la película es una bienintencionada y moralizante fábula de la factoría Disney sobre la persistencia, el valor y los buenos sentimientos, que incluye la conversión a mejores causas del desalmado periodista Harry Kingsley (Kevin Spacey).

En un entorno más contemporáneo, Natural Born Killers (Asesinos natos), (Oliver Stone, 1994), refleja la otra cara de este poder de los medios para generar figurar heroicas, a través del encumbramiento de dos asesinos en serie que se convierten en auténticos ídolos de masas. La servidumbre a las audiencias, la falta de escrúpulos de los profesionales de la comunicación y la propia esencia del medio televisivo se aúnan para hacer posible la sinrazón que plantea la película. El montaje final, que alterna un zapping por distintos canales televisivos con imágenes fragmentadas de la violencia generada por los asesinos y las alucinaciones relacionadas con ella, funciona como resumen final de la reflexión que la película hace en torno a la cultura fragmentaria y superficial propiciada por el medio televisivo.

Por otra parte, son muchas las películas de los 90, como Mars Attack! (¡Marte ataca!), (Tim Burton, 1996), Shadow Conspiracy (Conspiración en la sombra), (George Pan Cosmatos, 1997) o las ya mencionadas The Pelican Brief o Deep Impact, en las que los poderes públicos se muestran conscientes del poder de los medios y de su función como principales mediadores entre ellos y la sociedad, de ahí que en muchos casos se vea cómo los políticos buscan el modo de presentar la noticia a los profesionales de la información para que éstos la transmitan del modo más favorable posible ante la opinión pública. Además, en Deep Impact se reflexiona también sobre la importancia de la cultura de la imagen generada por los medios audiovisuales. De este modo, en la presentación del grupo de heroicos astronautas que tienen como misión salvar al planeta del impacto del cometa, el veterano capitán Spurgeon 'Fish' Tanner (Robert Duvall) expresa, en privado, su preocupación por la falta de conciencia del peligro que parecen tener sus compañeros. "Well, they're not scared of dyin'. They're just scared of looking bad on TV" (Deep Impact, 00:35:34), replica su interlocutor. El contraplano muestra a los pilotos posando sonrientes ante los medios, que les han rodeado sin descanso desde su primera aparición.

Esta omnipresencia mediática, unida a la guerra de las audiencias pareja con la proliferación de medios, conduce también a la espectacularización de la información,

6 "Bueno, no les asusta la muerte. Sólo temen quedar mal en la tele" (Deep Impact, 00:35:34). 
un fenómeno que ha llevado a los analistas a acuñar el término "infoentretenimiento" para referirse a los programas televisivos que mezclan la información con el entretenimiento y en los que resulta difícil discernir las fronteras entre ambos:

A number of scholars have argued that infotainment is incompatible with the needs of a democracy, degrading the quality of public information, dissuading from critical inquiry, and transforming rational argument into emotive spectacle (Baym, 2008, p. web ${ }^{7}$ ).

La denuncia de este fenómeno en el cine de los años 90 se evidencia de forma mucho más expresa en la producción española que en la norteamericana. De hecho, constituye el tema central de la representación del periodismo en el cine en España en dicha década, lo que sin duda tiene que ver con el nacimiento en 1990 de las primeras emisoras privadas de televisión y, con ellas, de un cambio sustancial en los modos de producción televisivos, mucho más orientados a partir de entonces hacia las audiencias después de un larguísimo periodo de monopolio de la televisión pública en España ${ }^{8}$.

De hecho, el límite entre lo que es información y lo que es entretenimiento no siempre queda claro en las películas españolas de la década. En Dame algo (Héctor Carré, 1997), la reportera televisiva Marisol Fernández (Nathalie Seseña) pasa de la información local al reality-show sin solución de continuidad. Lanzagorta (Javier Gurruchaga) presenta en Siempre hay un camino a la derecha (José Luis García Sánchez, 1997) un show televisivo presuntamente basado en hechos reales en el que la verdad se falsea y distorsiona sin pudor en busca de una mayor audiencia. El mundo de los reality shows y el nuevo universo televisivo surgido de la competencia entre cadenas constituye también el tema central de otras películas de la década, (no incluidas en el análisis por no guardar una relación directa con el periodismo y la información de actualidad) como Mamá es boba (Santiago Lorenzo, 1997), El grito en el cielo (Dunia Ayaso y Félix Sabroso, 1998) y Una chica entre un millón (Alvaro Sáenz de Heredia, 1993).

Uno de los retratos más destacados y extremos dentro a crítica del cine español de los 90 a la espectacularización de la información es Andrea Caracortada (Victoria Abril) en Kika (Pedro Almodóvar, 1994). Andrea es la directora de Lo peor de la semana, un espacio que recoge noticias de sucesos pero cuyo formato es más propio de un programa de entretenimiento que de un informativo.

En cuanto al cine norteamericano, la espectacularización de la información se aborda de manera expresa en To Die For (Todo por un sueño), (Gus Van Sant, 1995), forma parte de modo más sutil de muchos filmes de la década, por ejemplo en la evolución profesional de Tally Atwater (Michelle Pfeiffer), en Up Close \&Personal (Íntimo y personal), (Jon Avnet, 1996) y se vincula con la presentación ficcionalizada de

7 Varios estudiosos han argumentado que el "infoentretenimiento" es incompatible con las necesidades de la democracia, al degradar la calidad de la información que recibe el público, disuadirlo de tomar una actitud de investigación crítica, y transformar los argumentos racionales en un espectáculo emotivo (Baym, 2008).

8 Sobre el tema pueden consultarse los siguientes libros y trabajos: La realidad como espectáculo: Reality Show en España, 1990-1994 (Izaguirre Sabin, 1997); Políticas de televisión: La configuración del mercado audiovisual (Giordano \& Zeller, 1999). 
hechos reales en películas como The Insider, tal y como apunta la interesante reflexión de Carl Sessions (Sessions Stepp, 2000, p. web) con la que cerramos este trabajo:

$\mathrm{Al}$ respecto son muy interesantes las consideraciones expuestas por Carl Sessions Stepp, redactor jefe de American Journalism Review, quien en un interesantísimo artículo en el que compara la imagen del periodismo ofrecida por The Insider frente a la aportada por All the president's men, indica cómo The Insider disfraza de defensa del periodismo serio y responsable lo que en el fondo no es más que "infoentretenimiento" (refiriéndose a la propia película en sí):

Somewhere during "The Insider", there is a derisive reference to "infotainment", the use of information to entertain. "The Insider", like most docudrama, is the essence of infotainment, blurring all sorts of lines between reality and invention in ways that viewers cannot possibly fathom.

For example, many characters in the movie bear real names -Bergman, Mike Wallace, whistleblower Jeffrey Wigand - but some names have been changed. Some characters, especially the ones playing Wallace and Don Hewitt, are groomed, outfitted and postured to look and act like their real-world counterparts. Some words and actions correspond faithfully to reality, but others are made up.

The problem is that viewers can't tell what is true, what is half-true, what is guesswork, and what is fantasy. This is fundamentally unfair to both the characters and to history.

It is, amazingly enough, even unfair to Big Tobacco. To my way of thinking, almost any imaginable definition of evil would cover the deliberate making and selling of a product that is addictive and deadly. So it is hard to work up sympathy for the tobacco industry.

But "The Insider" romanticizes its journalist heroes so much and turns tobacco executives into such cardboard villains that the whole enterprise borders on caricature9. (Sessions Stepp, 2000, p. web).

9 En un momento de The Insider hay una referencia desdeñosa al "infoentretenimiento", el uso de la información para entretener. The Insider, como la mayoría de los docudramas, es la esencia del "infoentretenimiento", desvaneciendo toda clase de fronteras entre la realidad y lo ficticio de un modo que los espectadores difícilmente pueden descifrar.

Por ejemplo, muchos de los personajes de la película tienen nombres de personajes reales Bergman, Mike Wallace, el confidente Jeffrey Wigand - pero algunos nombres se han cambiado. Algunos personajes, especialmente los de Wallaces y Don Hewitt, están preparados, caracterizados y equipados para parecerse y actuar como sus equivalentes en la vida real. Algunas palabras y acciones corresponden fielmente a la realidad, pero otras no. El problema es que los espectadores no pueden saber qué parte es verdad, cuál es verdad a medias, cuál es un ejercicio de adivinación y cuál es fantasía. Lo cual es fundamentalmente injusto tanto para los personajes como para la historia. Es, bastante sorprendentemente, incluso injusto con Big Tobacco. A mí modo de ver, cualquier definición imaginable del mal incluiría hacer y vender deliberadamente un producto adictivo y mortal. Así que es difícil tenerle simpatía a la industria tabaquera. Pero la forma en que The Insider presenta al periodista como un héroe romántico y a los ejecutivos de la industria del tabaco como villanos de tebeo, hace que todo el empeño roce la caricatura. (Sessions Stepp, 2000) 


\section{Conclusiones}

La imagen de los medios de comunicación ofrecida por el cine de los años 90 es bastante consistente y reiterativa lo que permite alimentar la idea de que el cine contribuye a reforzar el imaginario del público con unos tópicos que se repiten de una película a otra. En lo que respecta a los manejados por las películas de los años 90, destaca la representación deshumanizada de los informadores. Si ya en décadas anteriores el cine sugería que los periodistas funcionan en manada (Vega Álvarez, 2003, p. 70), al final del siglo la manada se vuelve mucho más despersonalizada y agresiva que en épocas anteriores en el que es tal vez el rasgo genérico más llamativo en la representación cinematográfica de los periodistas. La enorme influencia de los informadores y sus medios en la vida de los ciudadanos, casi siempre en negativo, es otro aspecto que aparece reflejado de manera insistente en el cine de la década. Por último, el tercer gran rasgo que define la representación cinematográfica del periodismo a finales del siglo XX es el desdibujamiento de las fronteras entre información y entretenimiento.

\section{Referencias bibliográficas}

AGUILAR, Carlos (2007): Guía del cine español. Madrid, Cátedra.

BAYM, Geoffrey (2008): Infotainment. In: DONSBACH, Wolfgang (ed): The International Encyclopedia of Communication, Blackwell, Blackwell Publishing.

BEZUNARTEA VALENCIA, Ofa; CANTALAPIEDRA, María José; y GENAUT ARRATIBEL, Aingeru (2010): "Vivir y relatar la historia: la imagen de los corresponsales de guerra en el cine". Revista Latina de Comunicación Social [online], en: http://www.revistalatinacs.org/10SLCS/actas_2010/aingeru.pdf

BEZUNARTEA VALENCIA, Ofa; COCA GARCÍA, César; CANTALAPIEDRA GONZÁLEZ, María José; et al. (2010): "El perfil de los periodistas en el cine: tópicos agigantados". Intercom - Revista Brasileira de Ciências da Comunicaçao. vol. 33, n.1, pp. 145-167, jan./jun, São Paulo.

BEZUNARTEA, Ofa; CANTALAPIEDRA, María José; COCA, César et al. (2007): Periodistas de cine y de ética. Ámbitos., pp.329-393.

BEZUNARTEA, Ofa; CANTALAPIEDRA, María José; COCA, César et al. (2007): "Si hay sangre, hay noticia: recetas cinematográficas para el éxito periodístico". Palabra Clave. 10 (2), pp. 61-74.

BEZUNARTEA, Ofa; CANTALAPIEDRA, María José; COCA, César et al. (2008): "So What? She's A Newspaperman and She's Pretty. Women Journalists in the Cinema". Zer. 13 (25), pp. 221-242.

BEZUNARTEA, Ofa; CANTALAPIEDRA, María José; COCA, César et al. (2008): "Divismo y narcisismo de los periodistas en el cine". Textual \& Visual Media: revista de la Sociedad Española de Periodística. 13 (25), pp.107-120.

DEAHL, Rachel: Journalism In the Movies, Examining Journalism In the Movies. [online]. [Accessed 5 de enero 2009]. En: http://mediacareers.about.com/od/mediamovies/a/MediaMovies.htm 
EHLERS, Wibke (2006): With Pad and Pencil: Old Stereotypes in a New Form? A comparison of the Image of the Journalist in the Movies from 1930-1949 and 19902004. University of Canterbury.

EHRLICH, Matthew C. (2004): Journalism in the movies. Illinois, University of Illinois Press.

FILMAFFINITY (2013): [online] http://www.filmaffinity.com/es/main.html

GARCÍA DE LUCAS, Virginia; RODRÍGUEZ MERCHÁN Eduardo; y SALES HEREDIA, Javier (2006): Cine entre líneas. Periodistas en la pantalla. Valadolid, 51 Semana Internacional de Cine.

GHIGLIONE, Loren (1991): The American Journalist: Fiction Versus Fact. [online]. [Accessed 2 Oct 2008]. http://www.ijpc.org/ghiglione.htm

GHIGLIONE, Loren and SALTZMAN. Joe (2002): Fact or Fiction: Hollywood Looks at the News. [online]. [Accessed 7 enero 2009]. http://www.ijpc.org/hollywoodlooksatthenews2.pdf

GIORDANO, Eduardo y ZELLER, Carlos (1999): Politicas de televisión: La configuración del mercado audiovisual. Barcelona, Icaria Editorial.

GOOD, Howard (1998): Girl Reporter: Gender, Journalism, and the Movies. Scarecrow, Lanham, MD.

HERMAN, Sarah (2004-2005): Hacks, heels and Hollywood. How accurately do recent film portrayals of women journalists reflect the working world of their reallife counterparts? BA(HONS), Multi-Media Journalism.

IMAGE OF JOURNALIST IN POPULAR CULTURE. 2013. IJPC database.

INTERNET MOVIE DATABASE. 2013. [online]: www.imdb.com

IZAGUIRRE SABIN, José Luis (1997): La realidad como espectáculo: Reality show en España 1990-1994. Madrid, Universidad Complutense de Madrid.

LAVIANA, Juan Carlos (1996): Los chicos de la prensa. Madrid, Nikel Odeon.

MALTIN, Leonard (2005): Leonard Maltin's Classic Movie Guide. New York, Signet, Penguin Books.

MALTIN, Leonard (2008): Leonard Maltin's 2009 Movie Guide. New York, Signet, Penguin Books.

MARTÍNEZ-SALANOVA SÁNCHEZ, Enrique (2011): Periodismo y medios de comunicación en el cine. [online]. [Accessed 12 Jul 2013]. En: http://www.uhu.es /cine.educacion/cineyeducacion/periodismo.htm

MCNAIR, Brian (2010): Journalists in film. Heroes and Villains. Edimburgh, Edimburght University Press.

MÍNGUEZ SANTOS, Luis (2012): Periodistas de cine. El cuarto poder en el séptimo arte. Madrid, T\&B Editores.

MINISTERIO DE EDUCACIÓN, CULTURA Y DEPORTES (2013): Base de datos de películas calificadas (salas cinematográficas). [online]. [Accessed $24 \mathrm{Nov}$ 
2008] En: http://www.mcu.es/bbddpeliculas/cargarFiltro.do?layout=bbddpeliculas\&cache $=$ init

MORGAN, Michael (2008): “Cultivation Theory”. [online]. [Accessed 15 Jul 2013]: doi: 10.1111/b.9781405131995.2008.x

NESS, Richard R. (1997): From Headline Hunter to Superman. A journalist filmography. Lanham (Maryland), Sarecrow Press.

OSORIO, Olga (2009): La imagen de la periodista profesional en el cine de ficción de 1990 a 1999. A Coruña, Universidade da Coruña.

PARDO ALARCÓN CASTELLÓN, Vicent (2000): "100 películas de cine para trabajar la televisión en el aula" Comunicar, vol. 14, pp.159-172. En pdf: http://www .redalyc.org/pdf/158/15801422.pdf

\section{Anexo: Películas de los años 90 en las que aparecen periodistas}

\begin{tabular}{l}
\hline 1990 \\
\hline Dr. M (Club extinction) (Dr. M). (Claude Chabrol, 1990). \\
\hline Disparate nacional (Disparate nacional). (Mariano Ozores, 1990). \\
\hline Teenage Mutant Ninja Turtles: Movie, The (Las tortugas ninja). (Steve Barron, 1990). \\
\hline A Show of Force (Bajo otra bandera). (Bruno Barreto, 1990). \\
\hline Die Hard II (Die Hard 2: Die Harder) (La jungla 2: alerta roja (La jungla de cristal 2). (Renny Harlin, \\
1990). \\
\hline The Bonfire of the Vanities (La hoguera de las vanidades). (Brian de Palma, 1990). \\
\hline Navy Seals (Navy Seals, comando especial). (Lewis Teague, 1990). \\
\hline 1991 \\
\hline Teenage Mutant Ninja Turtles II: Secret of the Ooze, The (Las tortugas ninja II- El secreto de los \\
mocos verdes). (Michael Pressman, 1991). \\
\hline Adeus Princesa (Adiós princesa). (Jorge Paixao Da Costa, 1991). \\
\hline L.A. Story (Tres mujeres para un caradura). (Mick Jackson, 1991). \\
\hline Tacones lejanos (Tacones lejanos). (Pedro Almodóvar, 1991). \\
\hline Dead again (Morir todavía). (Kenneth Branagh, 1991). \\
\hline Cómo ser mujer y no morir en el intento (Cómo ser mujer y no morir en el intento). (Ana Belén, \\
1991). \\
\hline Year of the Gun (El año de las armas). (John Frankenheimer, 1991). \\
\hline 1992 \\
\hline The Public Eye (El ojo público). (Howard Franklin, 1992). \\
\hline A River Runs Through It (El río de la vida). (Robert Redford, 1992). \\
\hline La plague (La peste). (Luis Puenzo, 1992). \\
\hline Newsies (La pandilla). (Kenny Ortega, 1992). \\
\hline Universal soldier (Soldado universal). (Roland Emmerich, 1992). \\
\hline Bob Roberts (Ciudadano Bob Roberts). (Tim Robbins, 1992). \\
\hline Hellraiser III: Hell on Earth (Hellraiser III: Infierno en la Tierra). (Anthony Hickox, 1992). \\
\hline Hero (Héroe por accidente). (Stephen Frears, 1992). \\
\hline
\end{tabular}




\begin{tabular}{|c|}
\hline 1993 \\
\hline Dispara (¡Dispara!). (Carlos Saura, 1993). \\
\hline The Pelican Brief (El informe pelícano). (Alan J. Pakula, 1993). \\
\hline The remains of the day (Lo que queda del día). (James Ivory, 1993). \\
\hline Born yesterday (Nacida ayer). (Luis Mandoki, 1993). \\
\hline Father Hood (El enemigo público no1... mi padre). (Darrell James Rood \\
\hline L'Arbre, le maire et la mediatheque (El árbol, el alcalde y la mediateca) \\
\hline Groundhog Day (Atrapado en el tiempo). (Harold Ramis, 1993). \\
\hline Kika (Kika). (Pedro Almodóvar, 1993). \\
\hline Trois couleurs: Bleu (Tres colores: azul). (Krzysztof Kieslowski, 1993). \\
\hline Searching for Bobby Fischer (En busca de Bobby Fischer). (Steven Zailli \\
\hline Rosa Rosae (Rosa Rosae). (Fernando Colomo, 1993). \\
\hline Sleepless in Seattle (Algo para recordar). (Nora Ephron, 1993). \\
\hline 1994 \\
\hline Todos los hombres sois iguales (Todos los hombres sois iguales). (Man \\
\hline Iron Will (Voluntad de hierro). (Charles Haid, 1994). \\
\hline $\begin{array}{l}\text { Interview with the Vampire: The Vampire Chronicles (Entrevista con } \\
\text { 1994). }\end{array}$ \\
\hline Natural Born Killers (Asesinos natos). (Oliver Stone, 1994). \\
\hline Street Fighter (Street Fighter, la última batalla). (Steven E. de Souza, 19 \\
\hline I love trouble (Me gustan los líos). (Charles Shyer, 1994). \\
\hline Prêt-à-Porter (Prêt-à-Porter). (Robert Altman, 1994). \\
\hline Cómo ser infeliz y disfrutarlo (Cómo ser infeliz y disfrutarlo). (Enrique \\
\hline The Paper (Detrás de la noticia). (Ron Howard, 1994). \\
\hline Before the Rain (Pred Dozdot) (Before the rain (Antes de la lluvia)). (M \\
\hline Of Love and Shadows (De amor y de sombra). (Betty Kaplan, 1994). \\
\hline Intersection (Entre dos mujeres). (Mark Rydell, 1994). \\
\hline Angels in the Outfield (Ángeles). (William Dear, 1994). \\
\hline Blankman (Blankman, mi hermano el chiflado). (Mike Binder, 1994). \\
\hline The Hudsucker Proxy (El gran salto). (Joel Coen, 1994). \\
\hline 1995 \\
\hline Hotel Sorrento (Hotel Sorrento). (Richard Franklin, 1995). \\
\hline Dolores Claiborne (Eclipse total). (Taylor Hackford, 1995). \\
\hline The Bridges of Madison County (Los puentes de Madison). (Clint Eastw \\
\hline La ley de la frontera (La ley de la frontera). (Adolfo Aristarain, 1995). \\
\hline To die for (Todo por un sueño). (Gus Van Sant, 1995). \\
\hline La flor de mi secreto (La flor de mi secreto). (Pedro Almodóvar, 1995). \\
\hline Thunderbolt (Pi li huo) (Operación Trueno). (Gordon Chan, 1995). \\
\hline Mighty Aphrodite (Poderosa Afrodita). (Woody Allen, 1995). \\
\hline
\end{tabular}




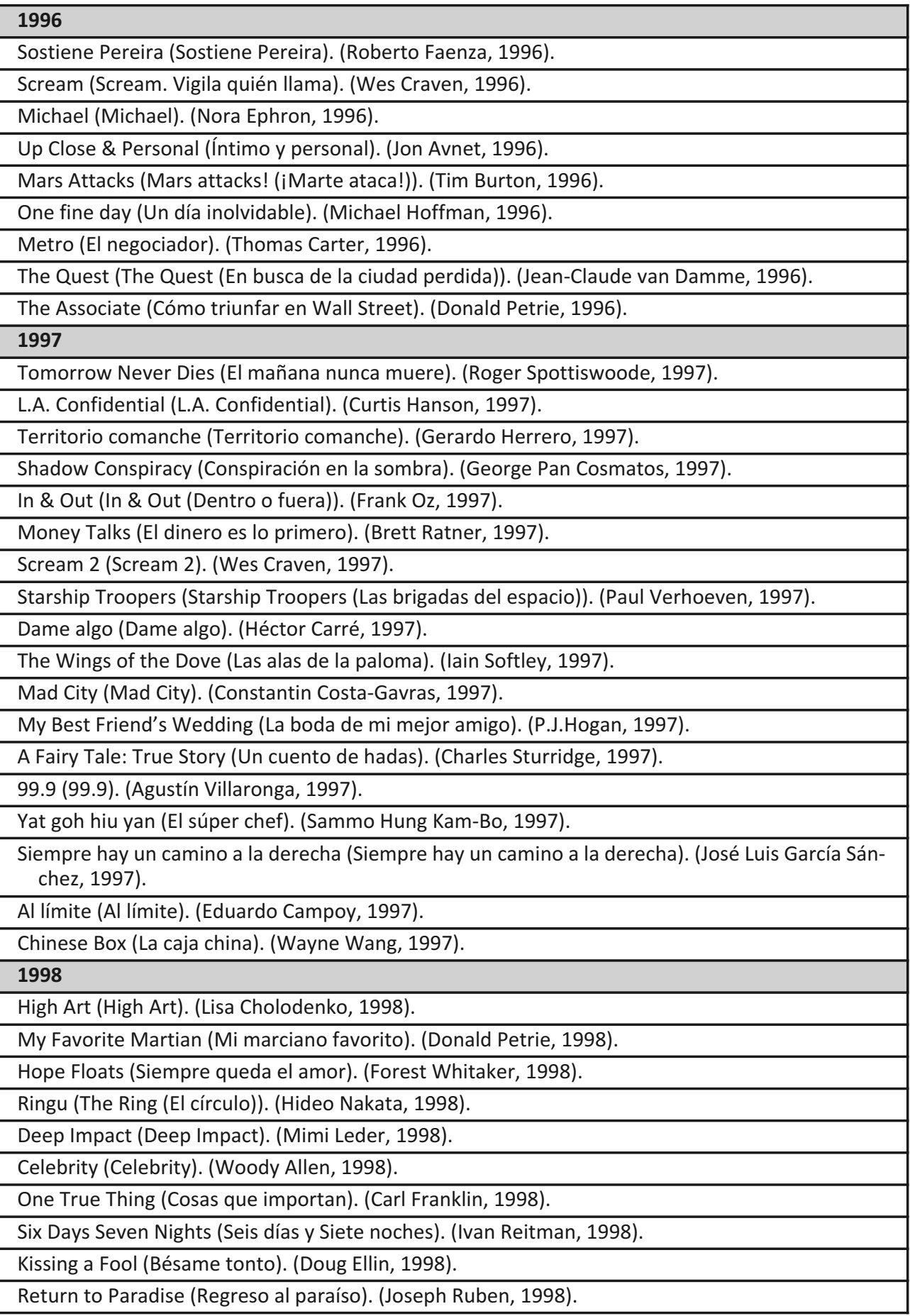




\begin{tabular}{|l|}
\hline Fear and Loathing in Las Vegas (Miedo y asco en Las Vegas). (Terry Gilliam, 1998). \\
\hline Velvet Goldmine (Velvet Goldmine). (Todd Haynes, 1998). \\
\hline 1999 \\
\hline Any Given Sunday (Un domingo cualquiera). (Oliver Stone, 1999). \\
\hline Three Kings (Tres reyes). (David O. Russell, 1999). \\
\hline The Big Brass Ring (La gran rueda del poder). (George Hickenlooper, 1999). \\
\hline Au coeur du mensonge (En el corazón de la mentira). (Claude Chabrol, 1999). \\
\hline Never Been Kissed (Nunca me han besado). (Raja Gosnell, 1999). \\
\hline Los sin nombre (Los sin nombre). (Jaume Balagueró, 1999). \\
\hline Runaway Bride (Novia a la fuga). (Garry Marshall, 1999). \\
\hline El mismo amor, la misma Iluvia (El mismo amor, la misma Iluvia). (Juan José Campanella, 1999). \\
\hline Nadie conoce a nadie (Nadie conoce a nadie). (Mateo Gil, 1999). \\
\hline Message in a Bottle (Mensaje en una botella). (Luis Mandoki, 1999). \\
\hline Snow falling on cedars (Mientras nieva sobre los cedros). (Scott Hicks, 1999). \\
\hline True Crime (Ejecución inminente). (Clint Eastwood, 1999). \\
\hline Tea with Mussolini (Té con Mussolini). (Franco Zeffirelli, 1999). \\
\hline RKO 281 (RKO 281. La batalla por “Ciudadano Kane”). (Benjamin Ross, 1999). \\
\hline The Insider (El dilema). (Michael Mann, 1999). \\
\hline Muppets from Space (Los teleñecos en el espacio). (Timothy Hill, 1999). \\
\hline
\end{tabular}

